The University of San Francisco

USF Scholarship: a digital repository @ Gleeson Library |

Geschke Center

2003

\title{
Feedback from the First Supernovae in Protogalaxies: The Fate of the Generated Metals
}

Keiichi Wada

Aparna Venkatesan

University of San Francisco, avenkatesan@usfca.edu

Follow this and additional works at: http://repository.usfca.edu/phys

Part of the Astrophysics and Astronomy Commons, and the Physics Commons

\section{Recommended Citation}

Keiichi Wada and Aparna Venkatesan. Feedback from the First Supernovae in Protogalaxies: The Fate of the Generated Metals. The Astrophysical Journal, 591:38-42, 2003 July 1. http://dx.doi.org/10.1086/375335

This Article is brought to you for free and open access by the College of Arts and Sciences at USF Scholarship: a digital repository @ Gleeson Library | Geschke Center. It has been accepted for inclusion in Physics and Astronomy by an authorized administrator of USF Scholarship: a digital repository @

Gleeson Library | Geschke Center. For more information, please contact repository@usfca.edu. 


\title{
FEEDBACK FROM THE FIRST SUPERNOVAE IN PROTOGALAXIES: THE FATE OF THE GENERATED METALS
}

\author{
KeIICHI Wada ${ }^{1,2}$ AND Aparna Venkatesan ${ }^{2,3}$ \\ Received 2002 December 2; accepted 2003 March 14
}

\begin{abstract}
We investigate the chemodynamic effects of multiple supernova (SN) explosions in the central region of primordial galaxies (e.g., $M \sim 10^{8} M_{\odot}$ halo at $z \sim 10$ ) using three-dimensional hydrodynamic simulations of the inhomogenous interstellar medium down to parsec scales. We find that the final protogalactic structure and metal distribution depend strongly on the number of SNe. Specifically, $1000 \mathrm{SNe}$ after an instantaneous burst of star formation are sufficient to almost completely blow away the gas in these systems, whereas $100 \mathrm{SN}$ explosions trigger the collapse of the protogalactic cloud, leading to the formation of a cold, dense clumpy disk $\left(n>300 \mathrm{~cm}^{-3}\right)$ with metallicity $Z=4 \times 10^{-4} Z_{\odot}$. These results imply that the metallicity of the "second generation" of stars could be $Z \sim 10^{-4} Z_{\odot}$, and that the environment to form metal-free stars in protogalaxies may be lost relatively quickly $\left(\$ 10^{7} \mathrm{yr}\right.$ ) after the first burst of $Z=0$ star formation. The recently discovered ultra-metal-poor star (Christlieb et al.) may represent surviving members of such second-generation star formation.
\end{abstract}

Subject headings: cosmology: theory — galaxies: formation — ISM: abundances —

ISM: kinematics and dynamics — ISM: structure — methods: numerical

\section{INTRODUCTION}

The prominent problems of cosmology and star formation have become increasingly intertwined in the last decade. This has been driven especially by studies of the feedback from the first stars and supernovae in high-redshift (z) protogalaxies on the intergalactic medium (IGM). The collapse of primordial gas in dark matter (DM) halos followed by the formation of the first metal-free stars has been extensively studied by several groups (e.g., Omukai \& Nishi 1998; Abel, Bryan, \& Norman 2000; Bromm, Coppi, \& Larson 2002). Examining the feedback from the first generation of supernovae $(\mathrm{SNe})$ is essential for understanding the metal enrichment and evolution of the interstellar medium (ISM), as well as the structure and subsequent star formation in the host protogalaxies. Some of the key physical issues in this problem are the propagation of metal-rich SN gas through the inhomogeneous ISM, and the subsequent mixing and diffusion of metals in the multiphase ISM. Mori, Ferrara, \& Madau (2002, hereafter MFM02), using threedimensional hydrodynamic simulations, found that a significant fraction of the metal-enriched gas in a $10^{8} M_{\odot}$ halo at $z=9$ is blown away, and that multiple SN explosions in primordial galaxies can play an important role in the metal pollution of the high- $z$ IGM. However, the early stages of the interaction between multiple blast waves from $\mathrm{SNe}$ in an inhomogeneous ISM are important for the subsequent evolution of the ISM in protogalaxies. From this point of view, the minimum resolved scale in MFM02-22 pc - is not fine enough to resolve the interactions between the blast waves and the clumpy ISM. Furthermore, self-gravity of the gas and radiative cooling below $10^{4} \mathrm{~K}$, both of which are not considered in MFM02, are crucial factors in determining

\footnotetext{
${ }^{1}$ National Astronomical Observatory of Japan, Mitaka, Tokyo 1818588, Japan; wada.keiichi@nao.ac.jp.

${ }^{2}$ CASA, UCB 389, University of Colorado, Boulder, CO 80309-0389.

${ }^{3}$ NSF Astronomy and Astrophysics Postdoctoral Fellow.
}

the internal structure of primordial galaxies after the initial $\mathrm{SN}$ explosions. This is especially relevant for cases in which the initial starburst is not strong enough to blow away the protogalactic gas.

In this paper, we report on the effects of an initial metalfree starburst in primordial galaxies, using three-dimensional hydrodynamic simulations with spatial resolution 3.9 pc, which take into account the self-gravity of the gas and radiative cooling between 20 and $10^{8} \mathrm{~K}$. We focus here on the simplest scenario, in which all the $\mathrm{SNe}$ are coeval and spatially concentrated; variations on these initial conditions will be considered in our future work. We explore feedback from relatively small numbers of $\mathrm{SNe}$ in order to see what kind of ISM structure and metal distribution are achieved in primordial galaxies just after the first $\mathrm{SN}$ explosions of firstgeneration stars. Studying SN feedback at these ISM scales has important consequences not only for the metal pollution of the high- $z$ IGM, but also for the duration of metal-free star formation in the early universe.

\section{NUMERICAL METHODS AND MODELS}

The hydrodynamic schemes that we use are described in Wada \& Norman (2001) and Wada (2001). We follow explicitly the diffusion of metals ejected from $\mathrm{SNe}$ into the ISM by solving the equation similar to that of mass conservation and assuming that the metal component has the same local velocity as the fluid. We calculate the gas cooling rate using cooling functions (M. Spaans 2002, private communication) for $20<T_{g}<10^{8} \mathrm{~K}$ and for gas metallicities in the range $Z=0-1 Z_{\odot}$. In the primordial gas, $\mathrm{H}_{2}$ is the main coolant below $10^{3} \mathrm{~K}$. The $\mathrm{H}_{2}$ and $\mathrm{HD}$ abundances are determined through a chemical network. The gas metallicity is a function of position and time. The radiative cooling for the gas in each grid cell is determined for the local metallicity (Goldsmith \& Langer 1978; Sutherland \& Dopita 1993; Spaans 1996). Note that in reality the CMB sets a physical 
temperature floor for the radiative cooling, e.g., $\sim 30 \mathrm{~K}$ at $z \sim 10$ (Bromm et al. 2002), although the gas temperature below $\sim 100 \mathrm{~K}$ is not very important for the evolution of the blast waves.

The hydrodynamic part of the basic equations is solved by AUSM (advection upstream splitting method). We used $512^{2} \times 128$ equally spaced Cartesian grid points covering a volume of $2 \mathrm{kpc} \times 2 \mathrm{kpc} \times 1 \mathrm{kpc}$. Therefore, the spatial resolution is 3.9 and $7.8 \mathrm{pc}$ for the $x-y$ and $z$ directions, respectively. The Poisson equation is solved to calculate the selfgravity of the gas using the FFT. The second-order leapfrog method is used for the time integration. We adopt the implicit time integration for the cooling term.

To achieve density and temperature distribution in a dark matter potential for the initial condition, we performed a pre-calculation in which a geometrically thin, dense disk is evolved in the spherically symmetric, time-independent DM potential, $\Phi_{\mathrm{DM}}$. We assumed $\Phi_{\mathrm{DM}} \equiv-(27 / 4)^{1 / 2} v_{c}^{2} /$ $\left(r^{2}+a^{2}\right)^{1 / 2}$, where $a=267 \mathrm{pc}$ is the core radius of the potential, and $v_{c}=11 \mathrm{~km} \mathrm{~s}^{-1}$ is the maximum rotational velocity. The mass of the dark matter in the central part of the protogalaxy is therefore $2 \times 10^{7} M_{\odot}$. The total mass and initial temperature of the gas are $10^{7} M_{\odot}$ and $20 \mathrm{~K}$. The gas is uniformly distributed in a thin axisymmetric disk, whose radius and scale height are, respectively, 400 and 8 pc. The total DM mass of $\sim 10^{8} M_{\odot}$ corresponds to a virial radius of $\sim 1 \mathrm{kpc}$ at $z \sim 10$ (MFM02; Madau, Ferrara, \& Rees 2001). Note that the very first stars could form in smaller systems of mass $\sim 10^{5}-10^{6} M_{\odot}$ at higher redshift $(z \sim 20-30)$, as studied in Bromm et al. (2002). Moreover, in the low-mass galaxies considered here, star formation followed by SNe might already have occurred during the initial collapse of the DM component. For simplicity, and owing to the short dynamical time scale of the gas component after the $\mathrm{SN}$ explosions, we assume here that the DM potential is time independent. A more self-consistent treatment will be considered in future work.

In the upper panels of Figure $1(t=0)$, the density and temperature distribution of the gas after virialization are shown. During the virialization process, the density distribution becomes almost spherical, although some dense clumps are formed in the central region where the temperature is less than $10^{3} \mathrm{~K}$. The mean density and temperature within $100 \mathrm{pc}$ from the center are $3 \mathrm{~cm}^{-3}$ and $100 \mathrm{~K}$. The total gas mass in the computing box is $5.6 \times 10^{6} M_{\odot}$. Gas in the cold phase $\left(T_{g}<100 \mathrm{~K}\right)$ occupies about $1.4 \%$ of the volume and has a mass of $1.9 \times 10^{6} M_{\odot}$. The maximum density in the dense clumps is $20 \mathrm{~cm}^{-3}$ (Jeans length $\sim 18 \mathrm{pc}$ ). We use this density, temperature, and velocity distribution as the initial condition.

In order to assess the chemodynamic effects of the first $\mathrm{SNe}$, some assumptions must be made about the initial mass function (IMF) of the first stars. There are significant uncertainties associated with this, since the processes that determine the stellar IMF, even in the local universe, are at present unclear. Some recent theoretical studies (e.g., Abel et al. 2000; Bromm et al. 2002) indicate that the primordial IMF may have been weighted toward masses exceeding $100 M_{\odot}$. If this were true, the metal yield per SN could potentially be much higher than that for the "usual" Type II SN. However, the duration and universality of the processes that lead to such an IMF are currently unknown. Given the unresolved nature of this topic, we assume a present-day Salpeter IMF over a stellar mass range of
1-100 $M_{\odot}$ in this work. We include metal generation only from $Z=0$ stars of mass 8-40 $M_{\odot}$ (Woosley \& Weaver 1995), assuming that stars more massive than $40 M_{\odot}$ implode directly into a black hole without mass/metal ejection into the ISM. For these assumptions, each unit mass of gas that forms stars produces total IMF-integrated masses in metals and stellar ejecta of 0.00663 and 0.1863 after the $\mathrm{SN}$ explosion. Furthermore, for the above assumptions, there is one SN for every $\sim 100 M_{\odot}$ of stars formed. Thus, the total metal yield and ejecta mass per SN on average are 0.663 and $18.63 M_{\odot}$, respectively. We input the energy $\left(10^{51}\right.$ ergs per SN) as thermal energy and mass/metal yields from the $\mathrm{SNe}$ at time $t=0$. We also explored cases in which the $\mathrm{SNe}$ are not coeval and occur over a period of $\sim 1 \mathrm{Myr}$. However, no essential differences from the models discussed in this paper were found.

Since the IMF and star formation efficiency in primordial galaxies are poorly understood, we take the number of $\mathrm{SNe}$, $N_{\mathrm{SN}}$, as a free parameter, and we explore two models here: model A $\left(N_{\mathrm{SN}}=10^{3}\right)$ and model B $\left(N_{\mathrm{SN}}=10^{2}\right)$. We put in $N_{\mathrm{SN}} \mathrm{SNe}$ randomly within the central $100 \mathrm{pc}$, since some of the initial star formation is expected in the dense, cold clumps seen in Figure $1(t=0)$. The energy and ejected masses in metals and gas from each $\mathrm{SN}$ are injected into a randomly selected grid cell, around which a blast wave is then expanded. This wave is not necessarily spherical, owing to the inhomogeneous density field and interactions with other $\mathrm{SNe}$.

A priori, we expect that if the total input energy is sufficiently larger than the binding energy, then the blast waves will blow the gas away, as has been predicted in many papers (e.g., Larson 1974 and references above). On the other hand, if the total SN energy is not efficiently converted into the ISM kinetic energy, the multiple blast waves would form a cavity in the protogalactic cloud. We would then expect the whole system to become dynamically unstable and collapse. This can be demonstrated in an analytic estimate as follows. Using a similarity solution of a blast wave in a radial density profile scaling as $\rho \propto r^{-2}$, the maximum radius of blast waves $r_{s}=(6 E / \bar{\rho})^{1 / 5} \tau_{c}^{2 / 5}$ for $\gamma=5 / 3$ (Ostriker \& McKee 1988), where $E$ is the input energy, $\bar{\rho}$ is the average density, and $\tau_{c}$ is the cooling time of the hot gas in the cavity generated by the $\mathrm{SNe}$. In our work here, $\tau_{c} \sim 2$ Myr, so that the cavity radius would be $\sim 200 \mathrm{pc}(\varepsilon / 0.1)^{1 / 5}$ $\left(N_{\mathrm{SN}} / 100\right)^{1 / 5}\left[\bar{\rho} /\left(0.03 M_{\odot} \mathrm{pc}^{-3}\right)\right]^{-1 / 5}\left(\tau_{c} / 2 \mathrm{Myr}\right)^{2 / 5}$, where $E=\varepsilon N_{\mathrm{SN}} 10^{51}$ ergs and $\varepsilon$ is the heating efficiency (the fraction of the total SN energy that is converted to the kinetic energy of the blast waves). When the hot gas in the cavity cools, we expect the protogalactic gas outside the cavity to lose pressure support and to begin falling toward the galactic center. The gas mass inside the cavity $\left(r<r_{s}=200 \mathrm{pc}\right)$ is about $10^{6} M_{\odot}$. If all the gas in the protogalaxy, about $5.6 \times 10^{6} M_{\odot}$, were to collapse and mix uniformly with the hot metal-enriched cavity, then the resulting average gas metallicity would be about $100 \mathrm{SN} \times\left(0.7 M_{\odot} / \mathrm{SN}\right) /$ $\left(6.6 \times 10^{6} M_{\odot}\right) \sim 5 \times 10^{-4} Z_{\odot}$.

In reality, the above estimates will be affected by the evolution of the multiple blast waves in an inhomogeneous medium, the fraction of protogalactic gas that eventually collapses, and by the heating efficiency, $\varepsilon$, which in this situation is unknown. In addition, the mixing of metals in the collapsing protogalaxy will be nonuniform. In our numerical method here, we do not have to make any specific assumptions for these factors. Instead, we explicitly solve 

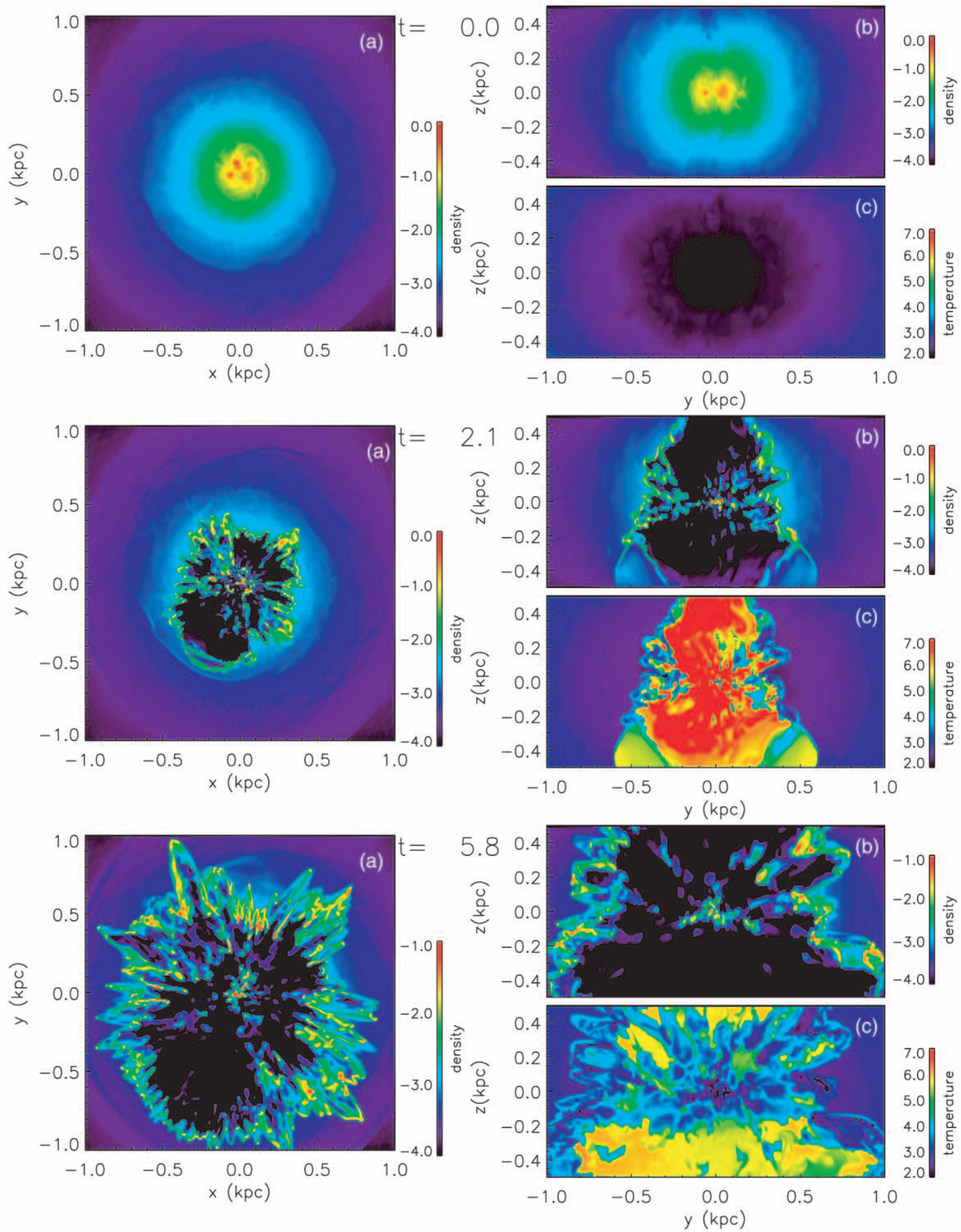

Fig. 1. - Plot of log-scaled density $\left(M_{\odot} \mathrm{pc}^{-3}\right)$ and temperature $(\mathrm{K})$ distributions of the initial condition $(t=0)$, and at two snapshots $(t=2.1$ and $5.8 \mathrm{Myr})$ of model A $\left(N_{\mathrm{SN}}=10^{3}\right)$. (a) The $x$ - $y$ cross sections of density. (b) The $y-z$ cross sections of density. (c) Same as $(b)$, but for temperature.

for the metal diffusion and the interaction between the multiple blast waves and the inhomogeneous ISM.

\section{RESULTS}

The evolution of the density and temperature distributions for model A are shown in Figure 1. The multiple SN explosions evolve as one big blastwave, which reaches the outer boundary of the $z$-direction at $t=2.1 \mathrm{Myr}$, and those in the $x$ - and $y$-directions at $t \sim 6 \mathrm{Myr}$. As seen in the figures, the explosions are roughly spherical, but dense "spikes" are formed, caused by nonlinear instabilities in the decelerated shocks (Yoshida \& Habe 1992). The hot gases in the cavity quickly cool from $\sim 10^{7} \mathrm{~K}$ at $t=2.1 \mathrm{Myr}$ to $\sim 10^{3}-10^{6} \mathrm{~K}$ at $t=5.8 \mathrm{Myr}$. At $t=3 \mathrm{Myr}$, the gas velocity is 2-4 times larger than the escape velocity in the whole region. This implies that $1000 \mathrm{SNe}$ are sufficient to blow away the gas in the protogalaxy in $\sim 10^{7} \mathrm{Myr}$. 

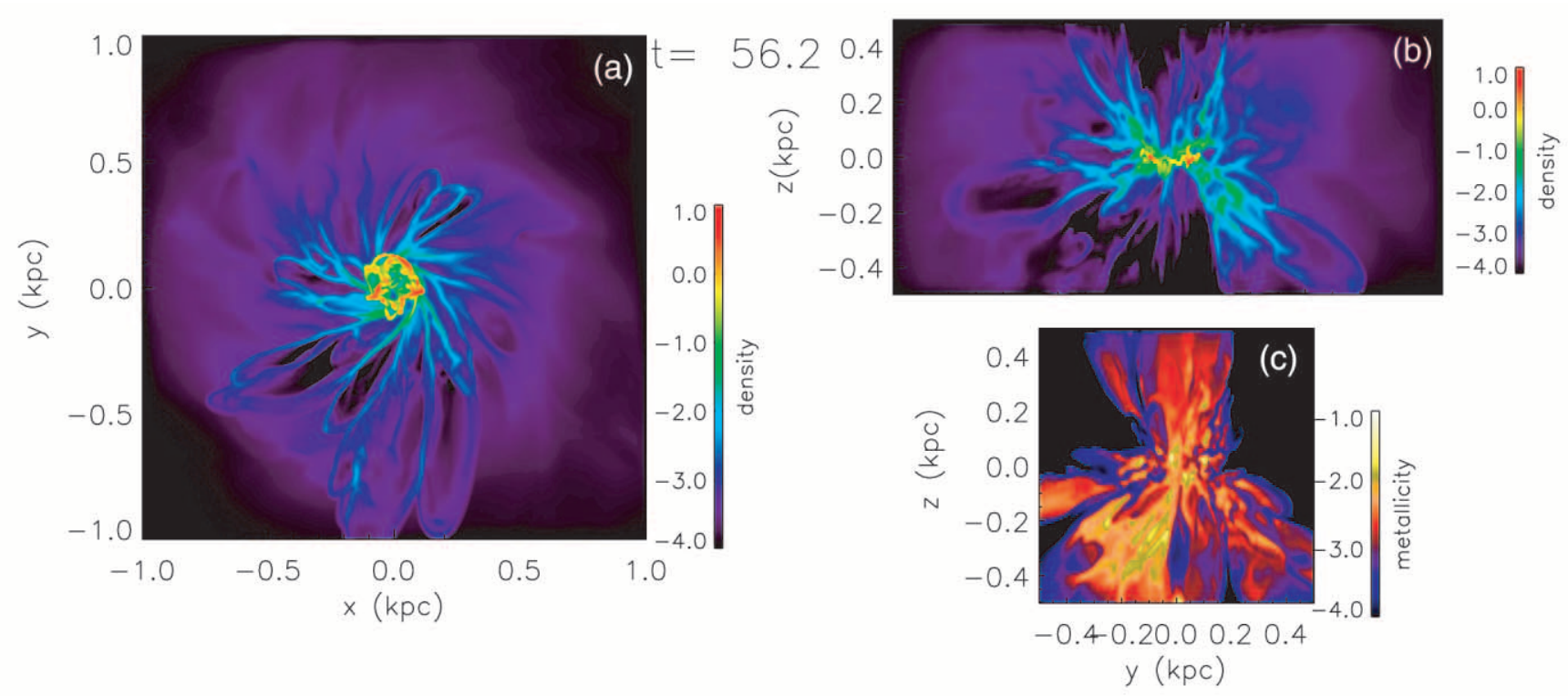

Fig. 2. - Same as Fig. 1, but for model B $\left(N_{\mathrm{SN}}=10^{2}\right)$ at $t=56.2 \mathrm{Myr}$. The lower right panel shows the $y-z$ cross section of the metallicity distribution in the central region. The plotted range of metallicities is $Z=10^{-4}$ to $10^{-1} Z$.

On the other hand, in model $\mathrm{B}$, the protogalaxy survives the initial starburst. The $100 \mathrm{SNe}$ generate a cavity in the central region of the protogalaxy, but it does not exceed sizes of $r \sim 300 \mathrm{pc}$. After $t \sim 20 \mathrm{Myr}$, the cavity collapses, which in turn triggers the collapse of the protogalactic gas cloud. Eventually a clumpy disklike structure, whose radius is about $200 \mathrm{pc}$, is formed in the center (Fig. 2). The $y-z$ cross section reveals a chimney-like structure. The diffuse gases seen in Figure 2 are still falling in toward the central dense disk. At $t=52.6 \mathrm{Myr}$, the mass of dense gas $\left(n>30\right.$ and $300 \mathrm{~cm}^{-3}$ ) is $1.6 \times 10^{6}$ and $7.2 \times 10^{5} M_{\odot}$, which are $36 \%$ and $16 \%$, respectively, of the total gas mass in the box at that time. The maximum density is $3.4 \times 10^{4}$ $\mathrm{cm}^{-3}$.

Figure $2 c$ shows that the metallicity distribution in the chimney is about $\left(10^{-3}\right.$ to $\left.10^{-2}\right) Z_{\odot}$, and that the diffuse halo is almost metal-free. The average metallicities in the very dense regions of $n>30$ and $300 \mathrm{~cm}^{-3}$ are $6.5 \times 10^{-4}$ and $4.1 \times 10^{-4} Z_{\odot}$. These are in contrast to $Z>10^{-2} Z_{\odot}$ in the relatively low density regions between the dense clumps in the central disky structure, owing to the formation of these clumps through the accretion of mainly metal-poor or metal-free gas.

As one may expect, the two models have different consequences for the global ejection of metals. Figure 3 shows the evolution of the radial metal mass distribution with time in the two models. Almost all the metals $\left(6 \times 10^{2} M_{\odot}\right)$ in model A are ejected out of the central protogalactic region, whereas most of the metals in model B return to $r<200 \mathrm{pc}$ in $50 \mathrm{Myr}$. As a result, the central region in model $\mathrm{B}$ is 10 times more metal rich than in model A.

In addition, the efficiency of converting the $\mathrm{SN}$ energy to the kinetic energy of the ISM is time-dependent. In model $\mathrm{B}$, this efficiency, defined as the ratio of the total ISM kinetic energy to total input energy, is 0.12 at $t=1 \mathrm{Myr}$ and $7 \times 10^{-3}$ at $t=10 \mathrm{Myr}$.
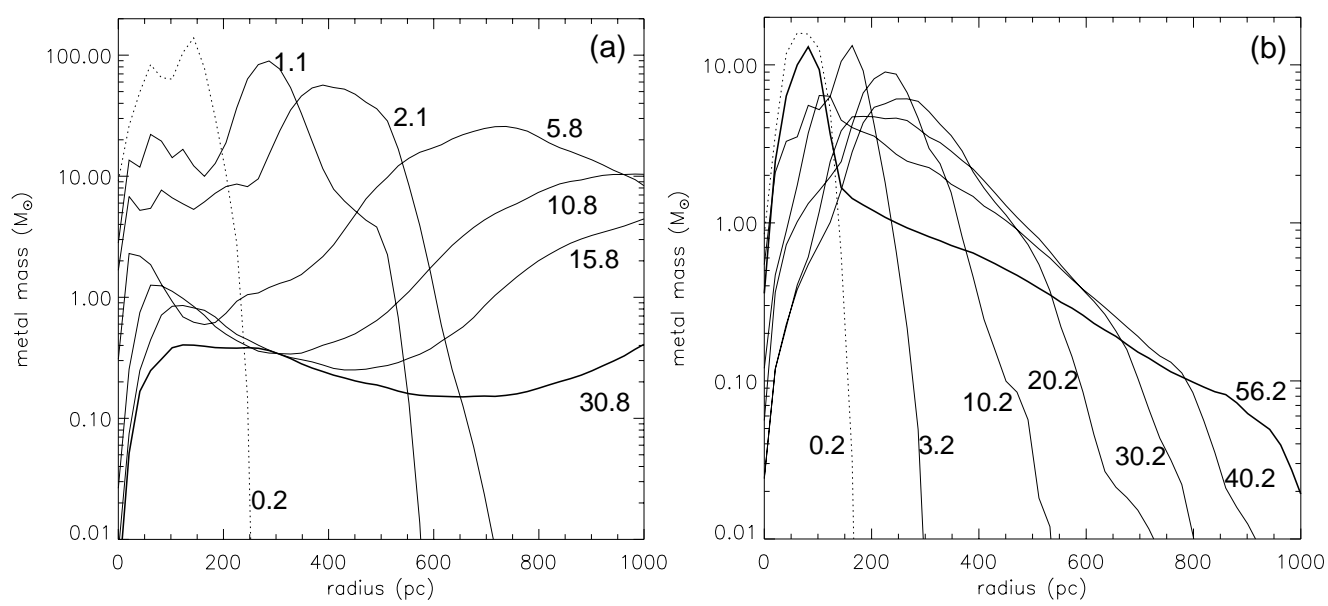

FIG. 3.-Time evolution of the radial distribution of metals in (a) model A and (b) model B. The individual curves are labeled with time in Myr. The metal mass is averaged over volume shells of width $20 \mathrm{pc}$ at each radius. 
In summary, our results in the context of the simplified models we have considered here- SN feedback from an instantaneous starburst in protogalaxies of mass about $10^{7}-$ $10^{8} M_{\odot}$ - are as follows. First, protogalaxies that undergo bursts with more than $10^{3} \mathrm{SNe}$ are not likely to retain the generated metals. The ultimate fate of the ejected gas and metals in this case is unclear, as they have not been tracked for sufficiently long timescales here. They may eventually escape to the IGM, or fall back partially to the galactic center, forming a dense core as found in the large dynamic range simulations by MFM02. Second, we find that less active starbursts $\left(N_{\mathrm{SN}} \sim 10^{2}\right)$ could lead to faster metal reincorporation in the gas, from which "second generation" stars with $Z \sim 10^{-4} Z_{\odot}$ could potentially form. The value of this transition metallicity is indicative rather than exact, given that we do not explicitly track the process of star formation itself, and that mixing processes depend on instabilities that may not be entirely resolved by our simulations. Nevertheless, this metallicity is interesting in terms of the recent discovery of an ultra-metal-poor star with $[\mathrm{Fe} / \mathrm{H}]=$ -5.3 (Christlieb et al. 2002). The abundance pattern of elements in this object suggests that it may have formed from a gas cloud that was pre-enriched by Type II SNe. Such a star could represent the surviving members of the secondgeneration stellar population in our scenario's model B, but with 10 times fewer initial $\mathrm{SNe}$. In addition, our derived value for the transition metallicity is in agreement with the numerical estimates in recent works (Bromm et al. 2001; Mackey, Bromm, \& Hernquist 2003; Schneider et al. 2002) for the critical level of enrichment that must occur in order for star formation in primordial gas to evolve from a hypothesized top-heavy stellar IMF to a present-day IMF. Future observations of ultra-low- $Z$ stars will strongly test these theoretical predictions for the metallicity and masses of second-generation stars.

\section{DISCUSSION}

In this paper, we have begun an investigation of $\mathrm{SN}$ feedback in high- $z$ protogalaxies. Our methods contain significant improvements over recent works, such as the inclusion of gas self-gravity in three dimensions and improved spatial resolution. These are necessary to quantitatively understand the effects of multiple SNe from the first stars at scales that are important for the ISM, which influences the cosmological relevance of early SNe for the IGM. We find that the fate of the generated metals - whether they are transported as a hot gas phase to the IGM or reincorporated quickly into cold star-forming gas in the protogalaxy-depends critically on the number of initial SNe. This has broad implications for the transition metallicity at early epochs from metal-free to second-generation star formation, and the rapidity of this process. The duration of metal-free star formation is of critical importance to theoretical studies of the $\mathrm{H}$ I and $\mathrm{He}$ II reionization of the IGM (see, e.g., Venkatesan, Tumlinson, \& Shull 2003), as well as for observational searches for such stars in the Galactic halo or at high redshift (Tumlinson, Shull, \& Venkatesan 2003, and references therein).

In the initial condition, the total mass of cold $\left(T_{g}<100\right.$ $\mathrm{K})$, dense $\left(n>3 \mathrm{~cm}^{-3}\right)$ gas is $3 \times 10^{5} M_{\odot}$. The gas mass converted to stars in model $\mathrm{A}$, with $10^{3} \mathrm{SNe}$, is about $10^{5}$ $M_{\odot}$. Therefore, if the star formation efficiency is larger than $\sim 0.3$, the first objects formed in the protogalaxies are almost pure stellar systems of mass $\sim 10^{5} M_{\odot}$, and the remaining baryonic mass is ejected from the galaxy. These metal-free stellar systems would not be bound, because a large fraction of the initial gas mass in the protogalaxy's central region is lost because of strong winds from the starburst's SNe. On the other hand, if the initial star formation efficiency is on the order of a few percent, the first starburst does not inhibit the subsequent creation of new star formation sites. If the star formation efficiency remains low, the metals from $\mathrm{SNe}$ cannot be expelled from the protogalaxies. In this case, metal-enriched stellar clusters of mass $\sim 10^{5}-10^{6} M_{\odot}$ eventually form, accompanied by a diffuse gaseous halo. These systems can be the building blocks of larger galaxies or the progenitors of globular clusters. In other words, suppose that the star formation efficiency is constant, e.g., 0.1 , among protogalaxies. The smaller systems $\left(M_{\text {total }}<\right.$ $\left.10^{7} M_{\odot}\right)$ would then dominate the metal enrichment of the IGM, which is consistent with the findings of Mac Low \& Ferrara (1999) and Ricotti, Gnedin, \& Shull (2002). The fate of the metals generated by the first stars is clearly linked closely to the mass and star formation histories of the host protogalaxies. In this work, we have considered a simple burst scenario in which the $\mathrm{SNe}$ were coeval and spatially concentrated, in order to focus on the ISM gasdynamics. We will examine the importance of varying these and other factors, such as the stellar IMF, in more detail in the future.

We are grateful to Andrea Ferrara and Massimo Ricotti for their useful comments, and for discussions on the simulations' initial condition. We thank the anonymous referees for helpful suggestions that improved the manuscript, and Marco Spaans for providing the gas cooling functions. Numerical computations were carried out on Fujitsu VPP5000 at NAOJ. A. V. gratefully acknowledges the support of NSF grant AST-0201670.
Abel, T., Bryan, G. L., \& Norman, M. L. 2000, ApJ, 540, 39

Bromm, V., Coppi, P. S., \& Larson, R. B. 2002, ApJ, 564, 23

Bromm, V., Ferrara, A., Coppi, P. S., \& Larson, R. B. 2001, MNRAS, 328, 969

Christlieb, N., et al. 2002, Nature, 419, 904

Goldsmith, P. F. \& Langer, W. D. 1978, ApJ, 222, 881

Larson, R. B. 1974, MNRAS, 169, 229

Mackey, J., Bromm, V., \& Hernquist, L. 2003, ApJ, 586, 1

Mac Low, M., \& Ferrara, A. 1999, ApJ, 513, 142

Madau, P., Ferrara, A., \& Rees, M. J. 2001, ApJ, 555, 92

Mori, M., Ferrara, A., \& Madau, P. 2002, ApJ, 571, 40 (MFM02)

Omukai, K., \& Nishi, R. 1998, ApJ, 508, 141

\section{REFERENCES}

Ostriker, J. P., \& McKee, C. F. 1988, Rev. Mod. Phys., 60, 1

Ricotti, M., Gnedin, N. Y., \& Shull, J. M. 2002, ApJ, 575, 49

Schneider, R., Ferrara, A., Natarajan, P., \& Omukai, K. 2002, ApJ, 571, 30

Spaans, M. 1996, A\&A, 307, 271

Sutherland, R. S., \& Dopita, M. A. 1993, ApJS, 88, 253

Tumlinson, J., Shull, J. M., \& Venkatesan, A. 2003, ApJ, 584, 608

Venkatesan, A., Tumlinson, J., \& Shull, J. M. 2003, ApJ, 584, 621

Wada, K. 2001, ApJ, 559, L41

Wada, K., \& Norman, C. A. 2001, ApJ, 547, 172

Woosley, S. E., \& Weaver, T. A. 1995, ApJS, 101, 181

Yoshida, T., \& Habe, A. 1992, Prog. Theor. Phys., 88, 251 